\title{
Numerical Observation of a Tubular Phase in Anisotropic Membranes
}

\author{
Mark Bowick, ${ }^{1}$ Marco Falcioni ${ }^{1}$ and Gudmar Thorleifsson ${ }^{2}$ \\ ${ }^{1}$ Physics Department, Syracuse University, Syracuse NY 13244-1130, USA \\ ${ }^{2}$ Fakultät für Physik, Universität Bielefeld, D-33615, Bielefeld, Germany
}

\begin{abstract}
We provide the first numerical evidence for the existence of a tubular phase, predicted by Radzihovsky and Toner (RT), for anisotropic tethered membranes without self-avoidance. Incorporating anisotropy into the bending rigidity of a simple model of a tethered membrane with free boundary conditions, we show that the model indeed has two phase transitions corresponding to the flat-to-tubular and tubular-to-crumpled transitions. For the tubular phase we measure the Flory exponent $\nu_{F}$ and the roughness exponent $\zeta$. We find $\nu_{F}=0.305(14)$ and $\zeta=0.895(60)$, which are in reasonable agreement with the theoretical predictions of RT $-\nu_{F}=1 / 4$ and $\zeta=1$.
\end{abstract}

PACS numbers: 64.60.Fr, 05.40.+j, 82.65.Dp 
Tethered membranes are 2-dimensional regularly triangulated surfaces fluctuating in 3 dimensions. Their behavior at thermal equilibrium is governed by the elastic and bending moduli, which correspond to the in-plane and out-of-plane rigidities respectively. Isotropic tethered membranes have been studied extensively, and it is known that they have a crumpled high temperature phase and a flat low temperature phase separated by a continuous phase transition, the crumpling transition [1] 3].

In a recent paper Radzihovsky and Toner (RT) have shown that anisotropy can radically change the nature of the phase diagram for tethered membranes [4, 5]. In particular a remarkable and completely unanticipated new phase of non-self-avoiding (phantom) tethered membranes - the tubular phase — is predicted. The tubular phase is characterized by the presence of long-range orientational order in one direction only - in the transverse directions it is crumpled. Furthermore it is expected that any degree of anisotropy will eliminate the direct crumpling transition from the flat to crumpled regimes and replace it by two transitions - a low temperature flat-to-tubular transition and a higher temperature tubularto-crumpled transition. Since it is very likely that real anisotropic tethered membranes can be observed experimentally, the rich structure of the phase diagram of these systems is exciting. There are several experimental realizations of anisotropic membranes one could imagine. Polymerized membranes with in-plane tilt order are good candidates [4]. It may also be feasible to cross-link in an applied electric field DNA molecules trapped in a fluid membrane [4]. Fluid membranes themselves also exhibit anisotropic "ripple" phases [6].

A tethered membrane in the tubular phase is quite different from real tubules, which are well defined cylindrical structures, such as lipid tubules, protein microtubules and carbon nanotubes [7 9]. Fluid membranes with chiral order exhibit tubular shapes [10]. In certain experimental conditions these tubules display a wealth of interesting behavior such as the pearling instability [11, 12].

The existence of an almost one-dimensional ordered phase is quite remarkable and, at least for phantom membranes, rather delicate. A tubular phase is possible because of the inevitable transverse stretching energy cost of bending fluctuations in the extended direction. Its existence is not trivial, though, because for physical tethered membranes, with internal dimension 2 and embedding dimension 3, the stability is marginal. The fluctuations of the tubule away from a straight linear shape (height fluctuations) along the extended direction are maximal with a corresponding roughness exponent of 1 [4]. For this reason it is imperative to check the existence of stable tubules with careful numerical simulations.

In this paper we establish the existence of tubules by large-scale Monte Carlo simulations of a discrete model of tethered membranes with the topology of a disk and free boundary conditions. The isotropic version of this model has been extensively studied by us recently 13 and so we are well-equipped to assess the effects of anisotropy.

We have chosen to implement the anisotropy in the bending rigidity rather than the elastic moduli of the model. Hence the membrane responds isotropically to in-plane stresses, but anisotropically to out-of-plane bending. This is conceptually clearer for us and, from the arguments given in [4], is equally good. Thus we choose the bending rigidity on links in the $x$-direction $\left(\kappa_{1}\right)$ of a triangular lattice to be larger than the bending rigidity along links in the other two directions $\left(\kappa_{2}\right)$. We study a system with aspect ratio one $\left(L_{x}=L_{y}\right)$, so that any anisotropy that develops is inherent to the system rather than introduced $a b$ initio. Different critical exponents arise [4] if one takes thermodynamic limits with other 


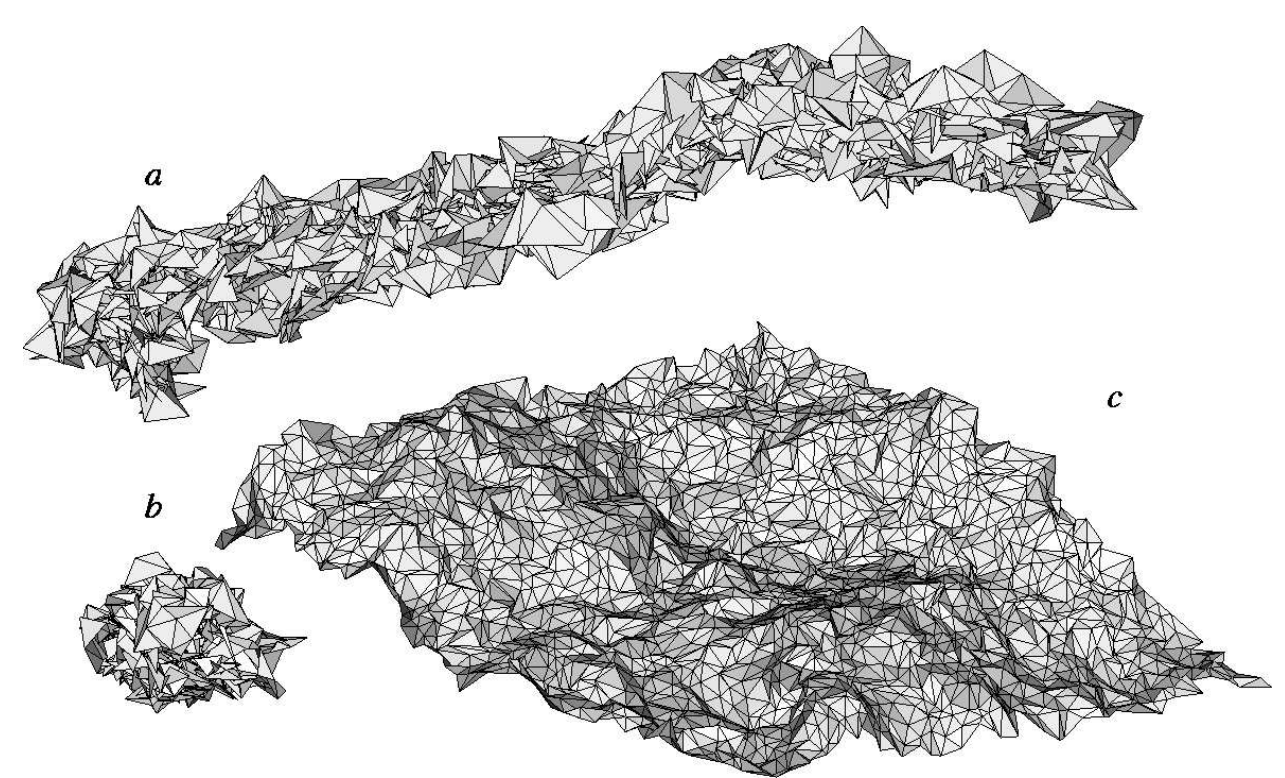

FIG. 1. a) An example of a tubular membrane. This is for $L=65$ and $\left(\kappa_{1}, \kappa_{2}\right)=(2,0)$. b) An isotropic membrane $(L=46)$ in the crumpled phase $(\kappa=0.5)$ and c) in the flat phase $(\kappa=1.1)$.

aspect ratios (i.e. if one tunes $L_{x}$ to diverge as some power of $L_{y}$ ).

By examining the specific heat along various paths in the $\left(\kappa_{1}, \kappa_{2}\right)$ plane we find that the model indeed exhibits two distinct transitions. The stronger transition is the one from the tubular to the flat phase. The tubular phase is characterized by equilibrium configurations which are extended in the $y$-direction and crumpled in the transverse direction (see Fig. 17a). Given this evidence for the existence of the tubular phase we proceed to measure the appropriate critical exponents and to compare them with the predictions of [4].

The exponents we focus on are the Flory exponent $\nu_{F}$ and the roughness exponent $\zeta$. The first gives the scaling of the transverse radius of gyration $R_{\perp}^{G}$ (the tubule radius) with system size $L, R_{\perp}^{G} \sim L^{\nu_{F}}$, and is predicted to be $1 / 4$. The second relates the height fluctuations in the extended $(y)$ direction to the system size $\left\langle h^{2}\right\rangle=\left\langle\left(h\left(x_{\perp}, L\right)-h\left(x_{\perp}, 0\right)\right)^{2}\right\rangle \sim L^{2 \zeta}$. The theoretical predictions of RT for these exponents for square phantom membranes are unambiguous. They find $\nu_{F}=1 / 4$ and $\zeta=1$. The maximal value for $\zeta$ corresponds to the previously mentioned marginal stability of the tubular phase.

Let us now give the details of our simulation. We analyze the partition function

$$
Z=\int[\mathrm{d} \mathbf{r}] \delta\left(\mathbf{r}_{\mathrm{cm}}\right) \mathrm{e}^{-\mathcal{H}[\mathbf{r}]}
$$

where $\mathbf{r} \in \mathbf{R}^{3}$ are the embedding coordinates and the delta function ensures that the center of mass motion is eliminated. The discrete Hamiltonian $\mathcal{H}$ is composed of a tethering potential, in the form of a Gaussian spring with vanishing equilibrium spring length, and (two) bending energy terms in the form of "ferromagnetic" interactions between nearest-neighbor normals $\mathbf{n}_{a}$ to the surface faces:

$$
\begin{aligned}
\mathcal{H}[\mathbf{r}] & =\sum_{\left\langle\sigma \sigma^{\prime}\right\rangle}\left|\mathbf{r}_{\sigma}-\mathbf{r}_{\sigma^{\prime}}\right|^{2} \\
& -\kappa_{1} \sum_{\langle a b\rangle}^{(x)} \mathbf{n}_{a} \cdot \mathbf{n}_{b}-\kappa_{2} \sum_{\langle a b\rangle}^{(y)} \mathbf{n}_{a} \cdot \mathbf{n}_{b} .
\end{aligned}
$$




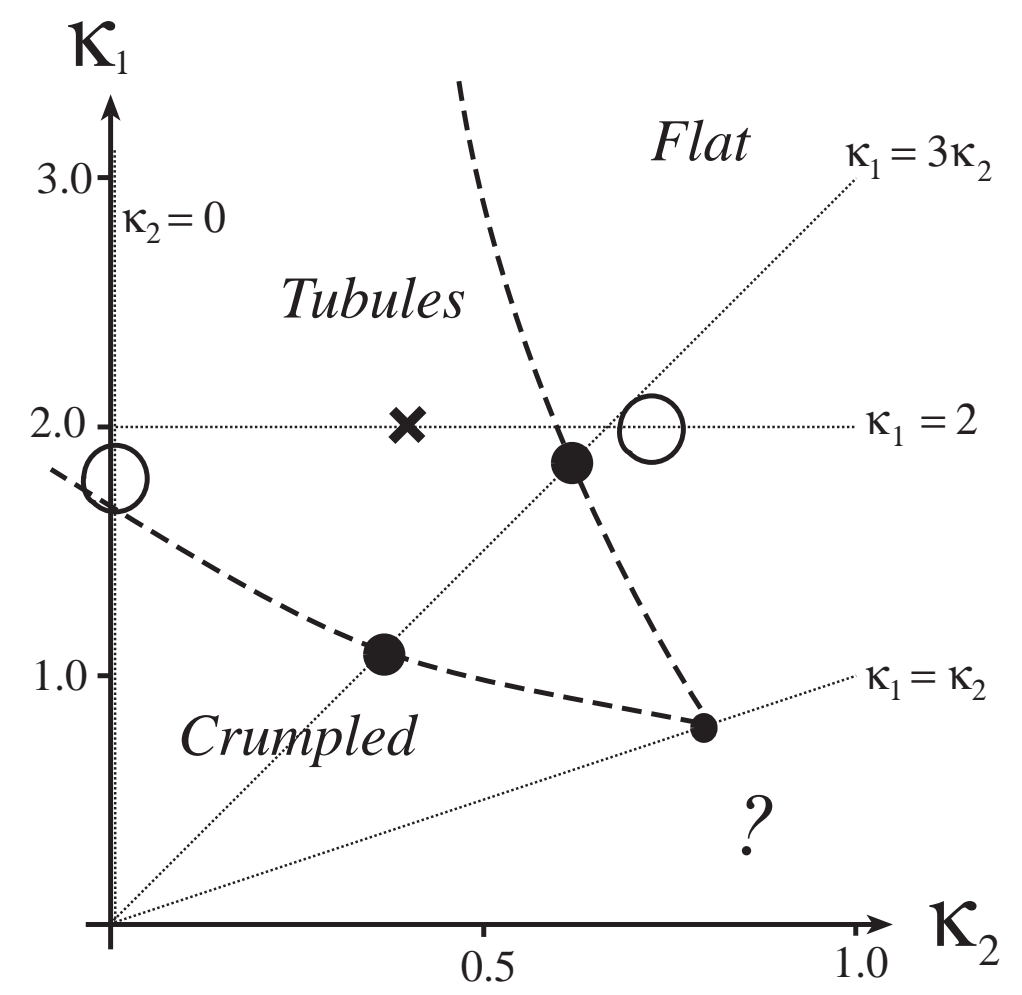

FIG. 2. The phase diagram of an anisotropic tethered membrane. The circles correspond to observed peaks in the specific heats $C_{V}^{x}$ and $C_{V}^{y}$ (the filled ones are from larger lattices). We performed simulations along the dotted lines with the cross indicating where we studied the tubular phase.

The second sum is over links in the $x$-direction and the third sum over the other two link directions. Note that no elastic constant are explicitly introduced; they are dynamically generated. We refer to [13] for an extensive treatment of the properties of this model for the flat phase of tethered membranes.

The Hamiltonian above was simulated using Monte Carlo methods for triangular lattices of up to $65^{2}$ nodes. The global shape of the lattice is chosen to be square with free boundary conditions. The field configurations $\{\mathbf{r}\}$ were updated using a unigrid algorithm, which substantially reduces the auto-correlation times compared to a simple Metropolis algorithm. We performed typically $10-20 \times 10^{6}$ sweeps per lattice volume and coupling — this resulted in about $5-10 \times 10^{3}$ independent measurements. The simulations required $\approx 50,000 \mathrm{CPU}$ hours on an IBM RS/6000 computer.

To explore the phase diagram predicted by RT we performed extensive simulations along the line $\left(\kappa_{1}, \kappa_{2}\right)=(3 \kappa, \kappa)$ (see Fig. 2). In addition we looked at the lines $(\kappa, 0)$ and $(2, \kappa)$ on smaller lattices. To look for evidence of a phase transition we measured the two bending energy terms in the action $E_{x}$ and $E_{y}$, for a range of couplings $\kappa$, and looked at their fluctuations or, more specifically, the two respective specific heats:

$$
C_{V}^{i}(\kappa)=\frac{\kappa^{2}}{L^{2}} \frac{\partial}{\partial \kappa}\left\langle E_{i}\right\rangle
$$




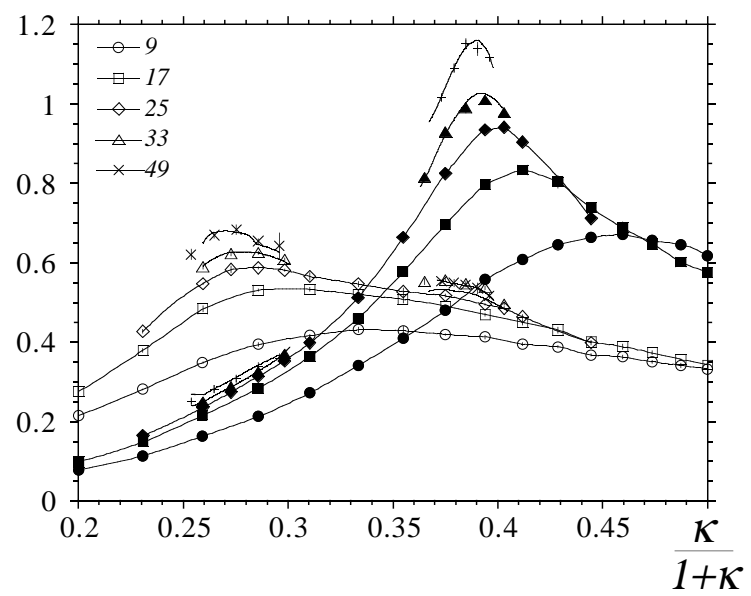

FIG. 3. The measured values of the specific heats $C_{V}^{x}$ (open symbols) and $C_{V}^{y}$ (filled symbols). The interpolating lines are obtained using multi-histogramming methods.

In Fig. 3 we show the measured values of $C_{V}^{x}$ and $C_{V}^{y}$ along the line $\kappa_{1}=3 \kappa_{2}$. This is for lattice sizes up to $49^{2}$. Both quantities show a divergent specific heat, characteristic of a phase transition. But what is remarkable is that the peaks occur at different values of the bending rigidity, signaling the existence of two distinct phase transitions. As the peak locations, which define the pseudo-critical couplings, shift as the volume is increased, one may naturally ask if they merge in the infinite volume limit. To check that, we have fitted the pseudo-critical couplings to the expected finite size behavior 114: $\kappa_{L}^{c}=\kappa_{\infty}^{c}+c / L^{1 / \nu}$. For the two peaks we get $\kappa_{\infty}^{c}=0.36(2)$ and $\kappa_{\infty}^{c}=0.62(2)$, respectively, convincingly excluding the possibility of them merging. The corresponding values of the exponent $\nu$ are $0.75(10)$ and 0.65(10), although we must caution that, for a reliable estimate, bigger lattices are needed. This in turn yields the critical exponent $\alpha$ governing the divergence of the specific heat. Assuming the validity of hyper-scaling, $\alpha=2-\nu d$, we find $\alpha \approx 0.5$ and 0.67 , consistent with a continuous phase transition.

One can also try to estimate $\alpha$ directly from the scaling of the peak heights, although that is more difficult. Preliminary estimates yield a small positive number, again somewhat smaller for $C_{V}^{y}$.

Taken at face value, this might indicate that the tubular-to-crumpled transition is somewhat weaker (slower divergence of the specific heat), although simulations on larger lattices are needed to confirm that the critical behavior of the two transitions really is different. This work is in progress. A detailed scaling theory of these two transitions has been developed in [5] and we plan to test this theory once we have the very high statistics required for such a comparison.

Performing a similar analysis along the other two lines in the $\left(\kappa_{1}, \kappa_{2}\right)$ plane, although on smaller lattices, yields the phase diagram shown in Fig. 2 15. This implies a three phase structure, the usual high-temperature crumpled and low temperature flat phases, together with the intermediate tubular phase predicted in [4].

To investigate the nature of this phase we have performed extensive simulations at the coupling $\left(\kappa_{1}=2, \kappa_{2}=0.4\right)$ on lattices ranging up to $65^{2}$ in extent. Direct evidence for the 


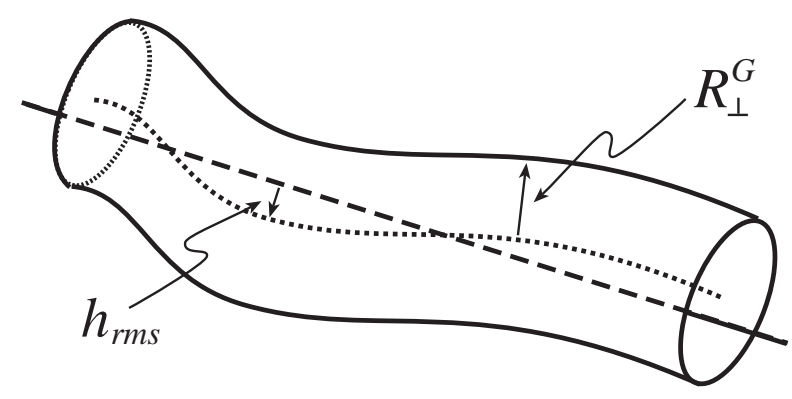

FIG. 4. The definition of the fluctuations of the zero-mode $h_{r m s}$ and the width of the tubule $R_{\perp}^{G}$. The dotted line indicates the one-dimensional shape of the tubule, whereas the dashed one is the optimal straight line.

tubular nature of the membrane is obtained by visual examination of equilibrium configurations (Fig. 11) and by measuring the scaling of the three eigenvalues of the shape tensor [13], defined as the off-diagonal part of the moment of inertia tensor. In a body-fixed frame these eigenvalues measure the extent of the membrane along the associated principal axes. We find that the largest eigenvalue scales as $L^{2 \nu_{F}}$, with $\nu_{F} \approx 0.988(11)$, while the other two have much smaller $\nu_{F}$. This indicates one extended direction and one crumpled direction i.e. a tubule.

A more detailed understanding of this tubular phase is obtained by looking at the fluctuations of the zero-mode of the tubule height $h_{r m s}$, analogous to the height fluctuations of a flat membrane, and the scaling of the width of the tubule $R_{\perp}^{G}$.

The fluctuations of the zero mode are defined as the deviations of the linear shape of the tubule from a straight line, whereas its width is the average distance of points from this onedimensional structure (Fig. 田). We have measured this width in two different ways. First, we fit both a polynomial, of sufficiently high degree, and a straight line to the membrane. Usually a polynomial of degree $\approx 15$ produces stable results. We then calculate the average distance of the polynomial from the straight line and the average distance of all points from the polynomial. This was done for about 10,000 independent configurations of the membrane for each of the lattice volumes $L=17,25,33,49$ and 65 (slightly fewer for the two largest volumes). The results were fit to the scaling predictions

$$
h_{r m s} \sim L^{\zeta} \text { and } R_{\perp}^{G} \sim L^{\nu_{F}}
$$

Discarding the smallest lattice size we find $\zeta=0.895(60)$ and $\nu_{F}=0.305(14)$. These values compare quite favorably with the analytic continuum predictions of RT: $\zeta=1$ and $\nu_{F}=1 / 4$. Thus the combined analytic and numerical investigations of tubules provide strong support for their existence and yield consistent values for critical exponents.

The second method we use is to slice the tubule up as a salami, defining a slice as the set of nodes having the same internal $y$-coordinate. Then the center of mass of the slice defines the linear shape of the tubule. If we consider the shape tensor of the ensemble of $\left\{x_{c m}\right\}$, the two larger eigenvalues scale as the width of the tubule (squared). We found that the two largest eigenvalues scale with Flory exponents $\nu_{F}=0.297(8)$ and $0.241(12)$, 
respectively. The smallest eigenvalue scales logarithmically with the volume $\left(\lambda_{\min } \sim \log (L)\right)$, which implies that the salami slices have vanishing thickness in the infinite volume limit, thereby justifying the use of the method. The scaling of the average distance of the c.m. of the salami slices from a straight line fit of the whole tubule yields the roughness exponent $\zeta=0.94(4)$, in reasonable agreement with the previously obtained value. It should be noted that the errors quoted are from the quality of the fit; the actual uncertainty is dominated by finite size effects.

In this paper we have established numerically for the first time the existence of the remarkable tubular phase of anisotropic tethered membranes predicted in [4]. Furthermore we measure the Flory exponent and the roughness exponent and find values not very far from those expected analytically. This should stimulate further work on the rich phase diagram of anisotropic tethered membranes.

There are two important directions to extend the simulations described in this letter. First one should investigate the anisotropic, rather than square membrane, scaling limits studied in [4,5]. For these systems the critical exponents are different. Even more exciting, perhaps, would be an investigation of self-avoiding anisotropic membranes. Self-avoidance causes extensive swelling of the membrane in the transverse direction and also lowers the roughness exponent $\zeta$ in the extended direction, as described by RT [4]. Since self-avoidance is irrelevant in the extended direction, in the tubular phase, it need only be implemented in the transverse direction. Such simulations should therefore be less demanding than analogous simulations for isotropic membranes. Precise predictions exist for all relevant exponents for self-avoiding anisotropic membranes in the tubular phase [4,5,16].

We would like to thank Leo Radzihovsky, Emmanuel Guitter and David Nelson for helpful discussions. We are grateful to NPAC (North-East Parallel Architecture Center) and the Cornell Theory Center for use of their computational facilities. The research of $\mathrm{MB}$ and MF was supported by the Department of Energy U.S.A. under contract No. DEFG02-85ER40237 and that of MF by a Syracuse University Graduate Fellowship. The research of GT was supported by the Alexander von Humboldt Stiftung and the Deutsche Forschungsgemeinschaft. 


\section{REFERENCES}

[1] D.R. Nelson, T. Piran, and S. Weinberg, editors. Statistical Mechanics of Membranes and Surfaces, volume 5 of Jerusalem Winter School for Theoretical Physics. World Scientific, Singapore, 1989.

[2] P. Ginsparg, F. David, and J. Zinn-Justin, editors. Fluctuating Geometries in Statistical Mechanics and Field Theory, Les Houches Summer School, Amsterdam, 1996. NorthHolland. http://xxx.lanl.gov/h94.

[3] Y. Kantor and D.R. Nelson, Phys. Rev. Lett., 58 (1987) 1289.

[4] L. Radzihovsky and J. Toner, Phys. Rev. Lett. 75 (1995) 4752 (cond-mat/9510172).

[5] L. Radzihovsky and J. Toner, University of Colorado report (to be published).

[6] T. Lubensky and F. Mackintosh, Phys. Rev. Lett. 71 (1993) 1565.

[7] B.P. Gaber, J.M. Schnur and D. Chapman eds., Biotechnological Applications of Lipid Microstructures 1988, Plenum, New York.

[8] H. Flyvbjerg, E. Jobs and S. Leibler, Proc. Natl. Acad. Sci. USA Vol. 93 (1996) 5975.

[9] M.S. Spector et. al., Proc. Natl. Acad. Sci. USA Vol. 93 (1996) 12943.

[10] J.V. Selinger and J.M. Schnur, Phys. Rev. Lett. 71 (1993) 4091.

[11] R. Bar-Ziv and E. Moses, Phys. Rev. Lett. 73 (1994) 1392.

[12] P. Nelson, T. Powers and U. Seifert, Phys. Rev. Lett. 74 (1995) 3384.

[13] M. Bowick. S. Catterall, M. Falcioni, G. Thorleifsson and K. Anagnostopoulos, J. Phys. I France 6 (1996) 1321 (cond-mat/9603157).

[14] To analyze the peaks we have interpolated between the measured couplings using multihistogramming methods. This was crucial in obtaining a reliable estimate of the location of the peak and the peak value. We are indebted to Kari Rummukainen for providing us with an appropriate code.

[15] Note that the triangular nature of the lattice we employ implies that the phase diagram is not symmetric about the isotropic point $\kappa_{1}=\kappa_{2}$.

[16] M. Bowick and E. Guitter, "Self-avoiding Tubules," Syracuse University and Saclay preprint SU-4240-660 and T97/036. 